\title{
Impact of a social media exercise service on individuals and employees
}

\author{
Matti Santtila ${ }^{1}$, Kalle Grönqvist² ${ }^{2}$ Jussi Räisänen², Heikki Kyröläinen ${ }^{1,3}$ \\ ${ }^{1}$ Department of Leadership and Military Pedagogy, National Defence University, Helsinki, Finland; ${ }^{2}$ H2Wellbeing, \\ Helsinki, Finland; ${ }^{3}$ Department of Biology of Physical Activity, University of Jyväskylä, Jyväskylä, Finland
}

\section{Summary}

Study aim: The purpose of the present study was to investigate the impact of a social media exercise platform (HeiaHeia, Helsinki, Finland) on the level of physical activity, physical fitness, wellbeing and body weight of the service users.

Material and methods: The subject group consisted of 2862 individuals who voluntarily participated in a web survey. Their age, gender, body mass index, physical fitness level and activity information were self-reported.

Results: Most of the service users (78.1\%) exercised more than three times a week. About $75 \%$ of the users reported that they were in good or excellent physical fitness, while about 50\% were overweight. More than half $(64.6 \%)$ of the service users reported that they had perceived an increase in their level of physical activity; and $46.4 \%$ of them reported that they had perceived an advance in their physical fitness after using the social media service. In addition, $54.0 \%$ of the users perceived an increase in their wellbeing. Every fifth (21.3\%) user reported a decreased body weight after using the service. Those users with lower levels of physical fitness, lower physical activity and who were overweight were more likely to report that the use of the present service was beneficial. In total, about $75 \%$ of the service users reported at least one benefit after using the service.

Conclusion: The present study demonstrated that the use of the social media exercise service can lead to an enhanced perceived level of physical activity, fitness and wellbeing. It also impacts positively on the users' body weight. Thus, the present social media service can be recommended for use, especially for overweight, unfit and sedentary customers.

\section{Keywords: Physical activity - Fitness - Overweight}

\section{Introduction}

Several investigations have confirmed that the current levels of daily physical activity and leisure-time physical activity are insufficient to maintain the health of citizens around the world $[2,4,8]$. A sedentary lifestyle is currently the fourth leading risk factor for premature mortality, and inactivity poses an even bigger threat to health than obesity $[4,23]$. Evidence indicates that a higher amount of total sitting time correlates with a higher risk of premature death [2]. Furthermore, a prolonged sedentary lifestyle appears to be a risk factor for all causes of mortality, even those independent of physical activity. According to Chau et al [2], each hour of daily sitting time is associated with a $2 \%$ increased risk for all causes of mortality. However, this association between the daily total sitting time and all causes of mortality was not linear. When adults sat for more than 7 hours per day, the risk factor increased by $5 \%$ for each 1-hour increment in the daily sitting time [2]. A high daily sitting time poses a health risk that is almost comparable with smoking, inactivity and obesity [19].
These factors have led to decreased levels of physical fitness and increased health problems associated with overweight persons in all age classes during the last decades $[7,15]$. Therefore, new innovations are needed to engage people in a physically active lifestyle. In this sense, the use of popular social media for the promotion of regular exercising could be an attractive option.

From a public health perspective, any method or means that increases daily physical activity is a useful one. Internet-delivered interventions for promoting physical activity and positive health-related behavioural changes and outcomes have become increasingly common across all age groups $[10,13,18]$. The association between social support - such as group chats, blogs, e-mail communication and social media - and greater physical activity has been reported in randomised controlled trial studies [3]. For example, Villiard and Moreno [21] noticed that Facebook is a popular discussion venue, particularly among adolescents. Fitness behaviour, physical activity and physical appearance were common topics in discussions among young people. However, these researchers found that using Facebook as a venue for distributing information did 
not increase the degree of physical activity among the young people when compared with conventional methods of health education [9]. Nonetheless, they concluded that distributing appropriate health and exercise related information using media in general, and social media in particular, would be fairly easy and beneficial [9]. In another study, Santtila et al [16] demonstrated that using a social media exercise service led to improved levels of perceived physical fitness. Furthermore, overweight, unfit and sedentary users seemed to benefit the most from these kinds of services [16]. However, the literature examining the effect of social media on promoting physical activity and fitness seems to be very limited in general, and in particular with regard to workplaces. The role of employers can be crucial for the promotion of the employee's wellbeing; and thus social media services could become a tool for promoting regular physical activity, although more research-based information is needed to confirm this.

Therefore, the aim of the present survey was to investigate the impact of a social media service on the users' selfestimation of physical fitness, physical activity, wellbeing and body weight. In addition, the present study also surveyed the daily sitting times and the impact of the service on the employees at some workplaces.

\section{Material and methods}

\section{Participants}

A total of 2862 users volunteered to take part in the survey, of whom 2083 (72.8\%) were women and 779 (27.2\%) were men. A total of 739 employees used the workplace platform that was offered by their employers. The largest age group of the respondents (26.2\%) was 31-40 years; while the smallest $(4.0 \%)$ one was the group under 21 years old.

\section{Protocol}

The web survey (provided by SurveyMonkey, Palo Alto, California, USA) was open to all service users of the HeiaHeia (H2 Wellbeing, Helsinki, Finland) web site for a period of one month. The background information of the users included their age group, gender, body mass index (BMI), self-estimated level of physical fitness and amount of physical activity. The survey also consisted of questions about the user's self-estimated changes in their degree of physical fitness, physical activity and wellbeing after using the social media service. To assess the level of physical activity, the survey asked the users to answer the following question (SIVAQ) [6]: "Think about the last three months. How many days a week, on average, did you exercise vigorously enough to cause increased breathing and perspiration?" All of the volunteers were informed about the design of the study, and by replying to the questionnaire they agreed to participate in the present study, which was conducted according to the 1975 Declaration of Helsinki.

The surveyed web service (platform) is an international and free-to-use social media exercise service (www. heiaheia.com) that is designed to motivate its users to be more physically active through encouragement from peers, friends and fellow employees. The service is based on social media interactions between the users and aims to promote their overall wellbeing, as well as functioning as a fitness promotion tool. The present survey participants voluntary signed in and utilised the web service as a tool to follow their daily physical activity and to get more information about proper training methods. In addition, employees at the workplaces were encouraged to utilise the web service by their employers.

\section{Data analysis}

For the statistical analysis, the frequency data was analysed by chi-square tests, and the single proportions were compared with z-tests. Additionally, the nonparametric Spearman correlation coefficients were calculated. The IBM SPSS 22.0 statistics package (IBM, CA, USA) was utilised, and the criterion of $\mathrm{p}<0.05$ was used for establishing the statistical significance.

\section{Results}

\section{Self-estimated physical activity, physical fitness and body mass index}

Most of the respondents (78.1\%) exercised more than three times a week (Fig. 1). A majority of them (52.8\%) exercised 3 to 4 times a week; whereas and $29.1 \%$ exercised twice or less. About $62.0 \%$ of the overweight users and $82.1 \%$ of the users with a normal body weight exercised for a minimum of three times a week. The self-estimated level of physical activity of the employees was on par with the other users (Fig. 1).

To survey the levels of physical fitness, the users were asked to self-estimate their fitness level on a scale ranging from poor to excellent. According to the scale, 5.1\% of the respondents answered excellent; $21.8 \%$ very good; and $50.0 \%$ good; while $23.0 \%$ of the participants estimated that they were in either the fair, poor or very poor category. Overall, the respondents in the normal weight group perceived themselves to be in better physical condition than the overweight respondents. The self-estimated level of physical fitness of the employees was on par with the other users (Fig. 2).

The body composition of the users was evaluated by surveying their BMI. About half of the respondents $(48.9 \%)$ were overweight or obese. More of the men $(56.0 \%)$ than the women $(43.1 \%)$ belonged to the overweight category. Also, fewer of the respondents who were 


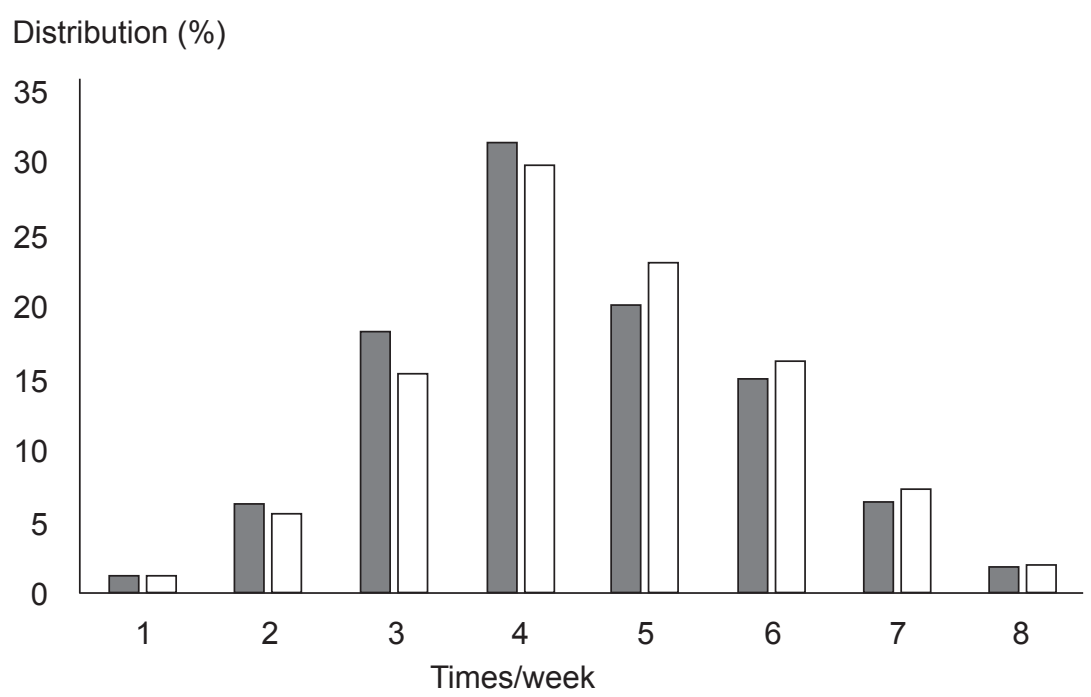

Fig. 1. Distribution of physical activity frequency of the responders, white bar all users and black bar workplace service users

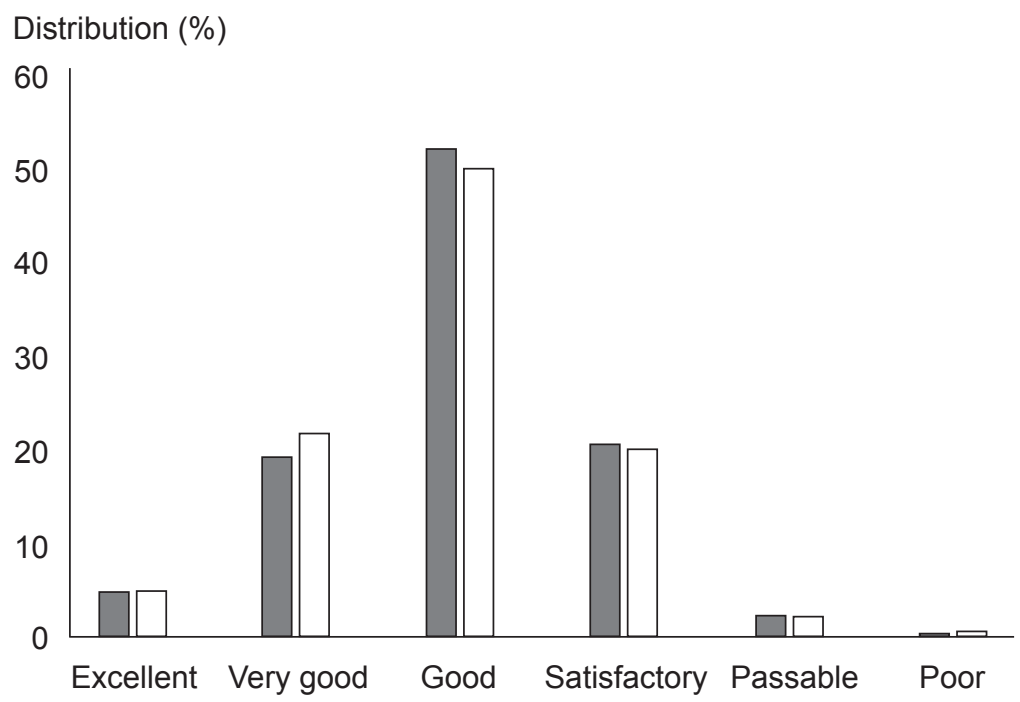

Fig. 2. Distribution of physical fitness categories of the responders, white bar all users and black bar workplace service users

under 30 years old were overweight $(27.2 \%)$ when compared to the respondents who were over 30 years $(51.2 \%)$. The prevalence of overweight respondents was highest $(56.1 \%)$ in the group that was over 50 years of age. The distribution of the employees by their BMI was on par with the other users.

\section{Impact of the service on the self-estimated physical fitness, physical activity, wellbeing and body composition}

Almost half of the respondents $(46.4 \%)$ reported an improvement in their physical fitness during the time when they used the web service. A slight majority (53.5\%) reported no noticeable impact. Also, about $40 \%$ of the employees reported that using the service had advanced their degree of physical fitness (Table 1). About half of the respondents (48.1\%), who had estimated their current level of fitness as fair or poor, reported an improvement in fitness while using the web service; as did $31.3 \%$ of the respondents in the excellent physical fitness category. In the overweight group (with a BMI over 25), 54.7\% responded that the use of the service had improved their perceived physical fitness; while the respondents with a BMI over 35 reported more positive effects than the respondents in any other body weight group (61.3\%). $45.6 \%$ of the physically active users (who exercised a minimum of 3 times/ week) responded that the use of the service had improved their perceived level of physical fitness; while $38.3 \%$ of those with a low level of activity (who exercised 0-2 times/week) reported a positive impact.

More than half of the individual respondents (64.6\%) reported that their perceived level of physical activity had 
Table 1. Impact of the use of social media service on self-estimated physical activity, fitness and wellbeing of all responders and employees $(\%)$

\begin{tabular}{lcccccc}
\hline \multirow{2}{*}{ Impact of service } & \multicolumn{2}{c}{ Significant increase (\%) } & \multicolumn{2}{c}{ Some increase (\%) } & \multicolumn{2}{c}{ No impact (\%) } \\
\cline { 2 - 6 } & All & Workplace & All & Workplace & All & Workplace \\
\hline Physical activity & 9.3 & 8.1 & 55.3 & 48.6 & 35.3 & 43.3 \\
Physical fitness & 3.9 & 3.2 & 42.5 & 36.0 & 53.5 & 60.6 \\
Wellbeing & 4.9 & 3.8 & 49.1 & 44.5 & 44.7 & 51.1 \\
\hline
\end{tabular}

increased due to the use of the web service (Table 1). Similarly, more than half of the employees $(56.7 \%)$ reported a respective increase. $68.4 \%$ of the overweight or obese users (with a BMI over 25) felt that their perceived degree of physical activity had increased after using the service; as opposed to $61.0 \%$ of the respondents in the normal weight category. In the fair or poor physical fitness category, $70.4 \%$ of the users reported a positive improvement in their physical activity due to the use of the service. In addition, $49.7 \%$ of the users in the excellent physical fitness category also reported a positive impact.

A slight majority of the individual respondents (54.0\%) reported an improvement in their sense of wellbeing as a result of using the service (Table 1). Almost half of the employees $(48.3 \%)$ responded that their perceived wellbeing had improved. Furthermore, $61.3 \%$ of the overweight and $49.4 \%$ of the normal weight users observed a positive impact. Almost half of the users in the fair or poor physical fitness category (48.1\%) reported that using the service had improved their sense of wellbeing; as opposed to $31.3 \%$ of the users with an excellent level of physical fitness. The physically active respondents who exercised a minimum of 3 times/week perceived more improvement in their sense of wellbeing due to their use of the service than the respondents with lower level of physical activity who exercised 0-2 times/week (51.3 and 47.7\%, respectively).

A fifth $(21.3 \%)$ of the service users reported a weight loss after using the service. The changes reported by the employees were on par with the respective changes by the individual users. Close to a third (30.5\%) of the overweight and obese users (with a BMI over 25) felt that using the service had led to a decreased body weight; as opposed to $14.4 \%$ of the users in the normal weight range. Nearly a third (32.1\%) of the users with fair or poor fitness reported weight loss; as did $12.9 \%$ of the users in excellent physical condition. Out of the physically active respondents (who exercised a minimum of 3 times/week), 20.7\% estimated that they had experienced a decrease in their body weight due to the use of the service. Additionally, in the lowest activity group (who exercised 0-2 times/week), $19.5 \%$ reported a weight loss.

To assess the daily sitting time, the users were asked to estimate how many hours a day they spent sitting, on average. $31.8 \%$ of the individual respondents sat for over eight hours a day; and $38.2 \%$ sat for less than five hours. The sitting times reported by the employees were $35.0 \%$ $(>8 \mathrm{~h} /$ day $)$ and $32.1 \%(<5 \mathrm{~h} /$ day $)$.

\section{The web service for workplaces}

The web service can also offer services designed specifically for employees at workplaces including but not limited to a web platform for tracking and charting their physical activities, rewards and incentives, and fitness challenges. The reported participation rates were high among the service users (91.2\%), and only a few (5.4\%) quit their company fitness challenges. Also, almost half of the respondents $(48.1 \%)$ felt that taking part in the individual web service for their workplace had improved their general alertness for physical activity.

The employees were asked: "What impact did the fitness challenge have on your workplace community?" The users were able to choose more than one option in their response (Fig. 3). The most widely reported effect was that exercise had become a more common topic for discussions at work, at that peer support fostered more activity. Only a few of the participants felt that the service caused a negative impact on their workplace community.

\section{Associations between physical fitness, physical activity, BMI and wellbeing}

After using the present service, the perceived level of physical activity was associated positively with the changes in the perceived degree of physical fitness $(r=0.44$, $\mathrm{p}<0.01)$, wellbeing $(\mathrm{r}=0.32, \mathrm{p}<0.01)$ and body weight $(\mathrm{r}=0.10, \mathrm{p}<0.01)$. An increase in the physical activity also had a positive impact on the perceived alertness $(r=0.26, p<0.01)$ among the employees. In addition, there was a weak association between the daily sitting time and the age, perceived degree of physical fitness, and physical activity of the respondents $(r=0.10-0.20, p<0.01)$, but there was no association with the BMI $(\mathrm{p}=0.451)$. Generally, the younger respondents sat for a shorter time than older ones. The respondents who were physically fit and active sat less, as a daily average, than the respondents who were less active and physically fit. 


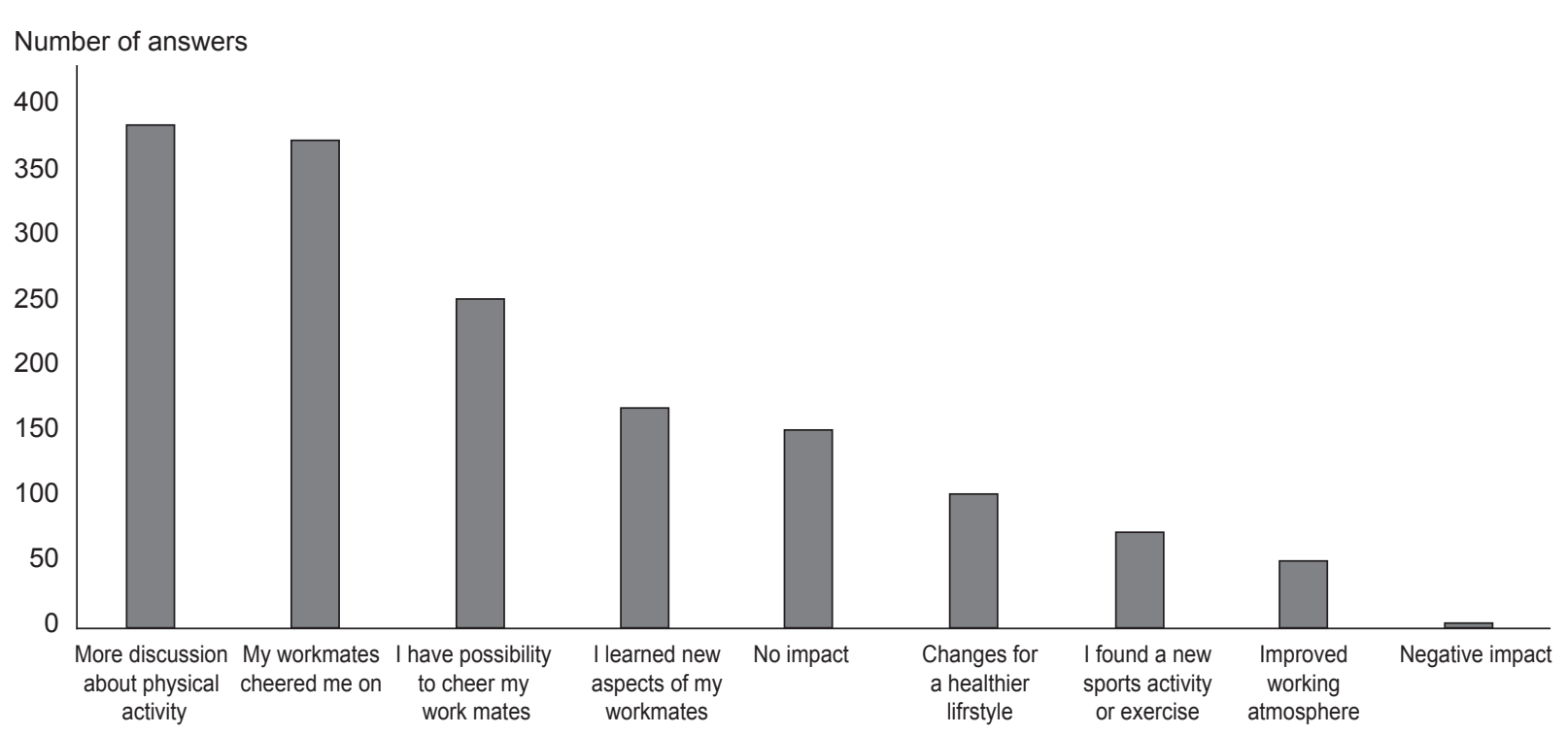

Fig. 3. Distribution of the responses of employees to the question: "What impact did the fitness challenge have on your work community?"

\section{Discussion}

The present study demonstrated that the social media exercise service had a positive impact on the users' perceived levels of physical fitness, physical activity and wellbeing. In addition, it helped the service users to control their body weight. Nearly half of the respondents reported that using the service improved their degree of physical fitness; and about two thirds of the respondents also reported an increased degree of physical activity. A slight majority of the users reported improvements in their perceived wellbeing due to the use of the service. Furthermore, one out of four respondents felt that using the service had led to a decrease in their body weight.

Using the web-based or mobile social media exercise service seemed to be especially beneficial for overweight users and users with lower levels of physical fitness. Namely, the overweight and unfit users were more likely to report an improvement in their degrees of physical fitness and an increase both in their amount of physical activity and sense of wellbeing. The overweight and unfit users were also more likely to experience a reduction in their body weight while using the service, as compared to the physically fit and active users. The service seems to be well-suited for users of all fitness levels, along with middle-aged and senior users. Alarmingly though, the smallest age group was comprised of the users under 21 years old, which is a group in particular need of encouragement to adopt a more active lifestyle [7, 15]. It is well-known that the levels of physical activity have decreased among young people and adolescents [7, 15]. In addition, a majority of the respondents were women, which reflects the overall gender distribution of the service users. The workplace service users generally reported the same positive improvements and/or impacts on their physical fitness, physical activity and sense of wellbeing as the individual users. This suggests that in the future, employers should invest in the development of services which especially target men and young people, so that they are also more likely to adopt and utilise these kinds of services.

A majority of the respondents exercised for a minimum of 3 to 4 times a week and estimated that their level of fitness was good or better. A third of the respondents exercised very little and self-estimated that their level of physical fitness was satisfactory. Thus, the respondents were slightly more physically fit and more physically active than the general population in Finland [12]. Reportedly, about half of Finns are sufficiently physically active to maintain their health [7]. In accordance with the general population, about half of the respondents were also overweight. According to the National FINRISK 2012 study, a slight majority of the adult Finnish population is either overweight or is obese [12]. It is worth mentioning that overweight users and those with lower levels of physical activity and fitness used the service regularly. Interestingly, the majority of the respondents were not so-called fitness enthusiasts, who might generally be widely expected to use these kinds of services. The national recommendation for health-enhancing physical activity is to participate in moderate-to-vigorous aerobic training 3 to 5 times a week. In addition, people should do strength and balance training 2 to 3 times a week [14]. Wantland et al [22] have previously suggested that web-based interventions increased the users resulting exercise times, as well as their 
knowledge of their nutritional status, asthma treatment and participation in healthcare, and also improved their body shape perception with a slower health decline during 18-months of weight loss maintenance. Thus, it seems to be important to promote the use of social media and other kinds of exercise-promoting web-services among inactive and overweight people.

In addition, there appears to be a social need for workplace services, which in the present study have reported an improvement in wellbeing, physical fitness and activity, and resulted in weight loss. A previous survey [16] also demonstrated the popularity of workplace fitness challenges, contests, tracking and charting of physical activities and supervised exercises. The surveyed service for workplaces enables employers to encourage their employees to pursue a more physically active workday with the support of exercises and activity challenges. The present survey also demonstrated that exercising became a more common topic of discussion in workplaces. Peer encouragement, in general, was considered to be a welcome and positive outcome of using the service, and only a very few of the employees felt that the service had a negative impact. A more active workday is also a useful tool for reducing the sitting time in offices.

One third of the respondents reported that they sat for more than seven hours a day. There was no association between the sitting time and the body weight. However, associations between the sitting time and the user's age, as well as the user's perceived physical fitness and amount of physical activity were observed. The younger service users and those who considered themselves as physically fit and active usually sat for shorter times than the other users. A sedentary lifestyle and sitting for prolonged periods are prevalent factors for growing lifestyle diseases and for premature death $[4,11,20]$. Regular exercise and health-enhancing physical activity can prevent cardiovascular diseases, Type 2 diabetes, musculoskeletal diseases, some cancers and obesity. Physical activity can also have a positive impact on a person's mental health, overall wellbeing and working ability $[1,4,5]$. Koster et al [8] recommended promoting more exercise and considerably reducing sedentary time as means of decreasing health risks. In addition, they called for recommendations on the maximum daily sitting time [8]. Furthermore, Ekelund et al [4] concluded that even a slight increase in the amount of physical activity can reduce health risks and the risk of premature death. As a response to these challenges, the social media exercise platform can offer a good, social and motivating service to promote the level of physical activity among less active people.

A limitation of the present study was that the respondents did not represent the whole population of the service users. Namely, the Finnish service users answered the survey on a voluntary basis. In addition, all of the changes in the surveyed parameters were self-estimated. For example, it is known that the amount of self-reported physical activity can often be overstated [6]. However, the present findings offer encouragement for further studies and the development of these kinds of services.

In conclusion, the present study demonstrated that the social media exercise service had a positive impact on the perceived levels of physical fitness, physical activity, wellbeing, and body weight among both the individual users and the employees. It should be emphasised that the benefits of using the service were more prominent among the unfit, sedentary and overweight users. This may also reflect positively on public health and healthcare expenses. Furthermore, the workplace service had a positive impact on the working environment and the employees' alertness to the need for daily physical activity. Finally, social media exercise services may provide a useful tool in motivating both individuals and employees to be more physically active.

\section{References}

1. Blair S.N., Kambert J.B., Kohl H.W., Barlow C.E., Macera C.A., Paffenbarger R.S. Jr, Gibbons L.W. (1996) Influences of cardiorespiratory fitness and other precursors on cardiovascular disease and all-cause mortality in men and women. JAMA, 276: 205-210.

2. Chau J.Y., Grunseit A.C., Chey T., Stamatakis E., Brown W.J., Matthews C.E., Bauman A.E., van der Ploeg H.B. (2013) Daily Sitting Time and All-Cause Mortality: A Meta-Analysis. PLOS ONE; 8: 11:e80000.

3. Cavallo D.N., Tate D.F., Ries A.V., Brown J.D., DeVellis R.F., Ammerman A.S. (2012) A social media-based physical activity intervention: a randomized controlled trial. Am. J. Prev. Med., 43: 527-532.

4. Ekelund U., Besson H., Luan J., May A.M., Sharp S.J., Brage S., etc. (2011) Physical activity and gain in abdominal adiposity and body weight: prospective cohort study in 288,498 men and women. Am. J. Clin. Nutr., 93: 826-835.

5. Erikssen G. (2001) Physical fitness and changes in mortality. The survival of the fittest. Sports Med., 31: 571-576.

6. Fogelholm M., Malmberg J., Suni J., Santtila M., Kyröläinen H., Mäntysaari M., Oja P. (2006) International Physical Activity Questionnaire: Validity against fitness. Med. Sci. Sports Exerc., 38: 753-60.

7. Husu P., Paronen O., Suni J., Vasankari T. (2011) Physical activity and fitness of Finns in 2010. The Ministry of Education and Culture. Publications: 15. ISBN 978-952263-034-6. [In Finnish].

8. Koster A., Caserotti P., Patel K.V., Matthews C.E., Berrigan D., Van Domelen D.R., Brychta R.J., Chen K.Y., Harris T.B. (2012) Association of sedentary time with 
mortality independent of moderate to vigorous physical activity. PLoS One, 7: e37696.

9. Leavy J.E., Rosenberg M., Barnes R., Bauman A., Bull F.C. (2013) Would you Find Thirty online? Website use in a Western Australian physical activity campaign. Health Promot. J. Austr., 24: 118-125.

10. Lustria M.L., Cortese J., Noar S.M., Glueckauf R.L. (2009) Computer-tailored health interventions delivered over the Web: review and analysis of key components. Patient Educ. Couns., 74: 156-173.

11. Matthews C.E., George S.E., Moore S.C. Bowles H.R., Blair A., Park Y., Troiano R.P., Hollenbeck A., Schatzkin A. (2012) Amount of time spent in sedentary behaviors and causespecific mortality in US adults. Am. J. Clin. Nutr., 95: 437-445.

12. Männistö S., Laatikainen T., Vartiainen E. (2014) Finnish obesity before and now. National FINRISKI 2012 study. National Institute of Health and Wellfare. ISBN: 978952-245-792-9. [In Finnish].

13. Norman G.J., Zabinski M.F., Adams M.A., Rosenberg D.E., Yaroch A.L., Atienza A.A. (2007) A review of eHealth interventions for physical activity and dietary behavior change. Am. J. Prev. Med., 33: 336-345. e316.

14. Physical Activity Pie. Physical activity guidelines (2009) UKK Institute. www.ukkinstituutti.fi/en. Based on Physical Activity Guidelines for Americans 2008: ODPHP Publication No. U0036.

15. Santtila M., Kyröläinen H., Vasankari T., Tiainen S., Palvalin K., Häkkinen A., Häkkinen K. (2006) Physical Fitness Profiles in Young Finnish Men during the Years 1975-2004. Med. Sci. Sports Exerc., 38: 1990-1994.

16. Santtila M., Grönqvist K., Räisänen J., Kyröläinen H. (2014) Impact on physical fitness of exercise promotion service utilizing social media. Biomed. Hum. Kinet., 6: 84-89.
17. Tammelin T., Ekelund U., Remes J., Näyhä S. (2007) Physical Activity and Sedentary Behaviors among Finnish Youth. Med. Sci. Sports Exerc., 39: 1067-1074.

18. Van den Berg M.H., Schoones J.W., Vliet Vlieland T.P. (2007) Internet-based physical activity interventions: a systematic review of the literature. J. Med. Internet Res., 9: e26.

19. Vasankari T. (2014) A generous sitting more risk of death. Finnish Medical Journal. 69: 25-32. [In Finnish].

20. Veerman J.L., Healy G.N., Cobiac L.J., Vos T., Winkler E.A., Owen N., Dunstan D.W. (2012) Television viewing time and reduced life expectancy: a life table analysis. Br. J. Sports Med., 46: 927-930.

21. Villiard H., Moreno M. (2012) Fitness on facebook: advertisements generated in response to profile content. $C y$ berpsychol. Behav. Soc. Netw., 15: 564-568.

22. Wantland D.J., Portillo C.J., Holzemer W.L., Slaughter R., McGhee E.M. (2004) The effectiveness of Webbased vs. non-Web-based interventions: a meta-analysis of behavioural change outcomes. J. Med. Internet Res., 6: e40.

23. WHO Library Cataloguing-in-Publication Data Global status report on noncommunicable diseases 2010 1. Chronic disease - prevention and control. 2. Chronic disease - epidemiology. 3. Chronic disease - mortality. 4. Cost of illness. 5. Delivery of health care. World Health Organization 2010. ISBN 9789241564229 (NLM classification: WT 500), ISBN 9789240686458.

\section{Received 03.02.2016 \\ Accepted 04.05.2016}

(C) University of Physical Education, Warsaw, Poland 